Alessandro Clemenzia

Janusz Aptacy

FTIC Florencja

DOI: $10.15290 /$ std.2015.01.02

\title{
ALCUNI SPUNTI SULL'ANTROPOLOGIA TEOLOGICA DI LUIS F. LADARIA
}

\section{SOME ASPECTS OF THE THEOLOGICAL ANTHROPOLOGY OF LUIS F. LADARIA}

This article tells about some aspects of the theological anthropology of Luis F. Ladaria, Spanish theologian, currently Secretary of the Congregation for the Doctrine of the Faith. Ladaria in his theological anthropology part horizons tracked by Vatican II and leads his reflection helped by various pronouncements of the Magisterium of the Church. It supports the outset that a theological anthropology must be determined by the Christology and the Trinity. Therefore, we need to have the mediation of Christ, who is true God and true man, the incarnate Word of God. This is not only in terms of concepts but also on the existence. It can be introduced in anthropological such as: love, communion, reciprocal. In this way you can go from the theological to the anthropological without the risk of applying to one of the features' another error in which has different theological reflections.

Key words: Ladaria, anthropology, hermeneutics, mediation, fliation, image, brotherhood.

\section{NIEKTÓRE ASPEKTY TEOLOGII ANTROPOLOGICZNEJ LUISA F. LADARII}

Niniejszy artykuł prezentuje niektóre aspekty antropologii teologicznej hiszpańskiego teologa Luisa F. Ladarii, obecnie Sekretarza Kongregacji Nauki 
Wiary. Teolog ten ukazując biblijne fundamenty antropologii teologicznej kładzie szczególny akcent na nauczanie Soboru Watykańskiego II na ten temat, a przede wszystkim na niektóre punkty Konstytucji duszpasterskiej o Kościele w świecie współczesnym Gaudium et spes. Ladaria prowadzi swoją refleksję teologiczno-antropologiczną wspierając się także innymi wypowiedziami Magisterium Kościoła. Utrzymuje on przede wszystkim, iż dla antropologii teologicznej determinującą jest chrystologia i trynitologia. Jest więc konieczną dla człowieka mediacja Chrystusa, prawdziwego Boga i prawdziwego człowieka, Wcielonego Słowa Bożego, nie tylko na poziomie pojęć, lecz także lingwistycznym i egzystencjalnym. Dzięki temu możliwe jest wprowadzenie do antropologii tematów takich jak: miłość, komunia, wzajemność; można też przechodzić, dzięki pośrednictwu Chrystusa, od wymiaru teologicznego do antropologicznego, bez ryzyka przypisywania jednemu charakterystyki drugiego, błędu, w który popadły rożne refleksje teologiczne.

Można stwierdzić, iż Ladaria jest typowym przedstawicielem teologicznej antropologii posoborowej, ukazującej tożsamość oraz pełną realizację człowieka jedynie w Chrystusie - Bogu Człowieku, nie zaś w takiej, czy innej kategorii socjologicznej.

Słowa klucze: Ladaria, antropologia, hermeneutyka, pośrednictwo, usynowienie, obraz, braterstwo.

\section{Introduzione}

Per presentare al meglio il contributo di mons. Luis F. Ladaria nell'ambito dell'antropologia teologica è necessario rintracciare quel peculiare punto di partenza del discorso che andrà a determinare l'articolazione di tutto il suo pensiero successivo. Si tratta di un punto iniziale che segna e caratterizza non solo il contenuto del suo pensiero, ma anche il suo stesso metodo interpretativo e processo argomentativo. Ma di quale "punto" si tratta, tanto da ricoprire una tale importanza? Quale è quell'elemento originale e originario che funge da bussola per l'antropologia?

Prima ancora di rispondere a queste domande, che portano al centro della riflessione, è bene mettere in luce alcune linee che introducono il tema, evidenziate dallo stesso Ladaria, per arrivare ad una migliore individuazione dell'oggetto materiale e di quello formale.

Quando si parla dell'uomo, soprattutto alla luce dell'odierno contesto socioculturale, non si può non tenere conto dei differenti piani semantici all'interno dei quali ci si muove. Ladaria afferma a tale proposito: «Si può parlare dell'uomo, e difatti si parla di lui, da molti punti di vista: filosofico, psicologico, medico, sociologico [...] il termine "antropologia" è diventato in molti casi un 
termine equivoco» ${ }^{1}$. Tale equivocità non significa che la riflessione teologica debba risentire di una certa ambiguità; l'oggetto materiale dell'antropologia teologica, infatti, è chiaro; afferma ancora Ladaria: "È evidente che questo vocabolo ci rimanda all'uomo, ci fa vedere che costui è l'oggetto materiale del nostro studio" ${ }^{2}$. Non basta, tuttavia, l'oggetto materiale; ancora più importante è avere chiaro l'oggetto formale dell'antropologia, vale a dire l'occhio prospettico attraverso il quale viene argomentato l'uomo: "Dobbiamo anche precisare, e questo è senza dubbio molto importante, il punto di vista da cui noi cerchiamo di affrontarlo. Laggettivo 'teologica' ci dice quale è questo punto di vista: si tratta di ciò che l'uomo è nella sua relazione con il Dio Uno e Trino rivelato in Cristo" ${ }^{3}$.

Ed è proprio dall'individuazione dell'oggetto materiale e dell'oggetto formale che Ladaria delinea un possibile significato di "antropologia teologica", vale a dire "quella disciplina [...] quella parte o settore della teologia dogmatica che ci insegna ciò che siamo alla luce di Gesù Cristo rivelatore di Dio"4.

Già da quest'ultima citazione si può intravedere come l'antropologia teologica contenga, all'interno della sua stessa natura, l'uomo, Cristo e Dio Trinità ${ }^{5}$. L'antropologia si fonda su questi tre precisi ambiti. Ma come articolarli tra loro? È nel rispondere a questa domanda che ci troviamo all'interno di quell'elemento originale e originario che funge da bussola per l'antropologia teologica.

Questa articolazione dell'antropologia con la cristologia e la trinitaria si rintraccia anche all'interno dello stesso lemma "persona", termine che, pur caratterizzando l'essere uomo, trova nel suo riferimento teologico e cristologico il suo senso più vero e autentico ${ }^{6}$.

\section{La necessaria mediazione cristologica}

Per rispondere alle domande precedentemente poste, si può affermare che soltanto "in Cristo" si può articolare un corretto e proporzionato rapporto tra

$1 \quad$ L. F. Ladaria, Introduzione alla antropologia teologica, Gregorian \& Biblical Press, Roma $2011^{7}$, p. 9. Dato che il presente articolo verrà pubblicato in Polonia, segnaliamo qui anche una pubblicazione in lingua polacca sul tema in questione: K. Góźdź, Chrysto-antropologia abpa Luisa F. Ladarii, „Studia Bobolanum” 1(2011), p. 17-27.

2 L. F. Ladaria, Introduzione alla antropologia teologica, op. cit., p. 9.

3 Ibidem.

4 Ibidem.

5 Per argomentare questo discorso, Ladaria ha come costante punto di riferimento il documento della Commissione Teologica Internazionale: Teologia - cristologia - antropologia, (10.1982), in Enchiridion Vaticanum 8, Bologna 20049', nn. 404-461.

6 Cfr. Antropologia teologica, Piemme, Casale Monferrato (Al) 2002³ , pp. 158-162. 
quei due misteri che si intrecciano sin dalla creazione del mondo: quello di Dio e quello dell'uomo. Per argomentare quanto affermato, è bene prima sottolineare una costante che compare negli scritti di Ladaria, tanto da caratterizzare la sua teologia: non si può affermare l'uomo senza affermare, contemporaneamente, Dio. Perché questa simultaneità non porti a un confuso rapporto tra il piano divino e quello umano, è necessaria la mediazione di Cristo, vero Dio e vero uomo; una mediazione sia in senso soteriologico sia in quello rivelativo. Soteriologico, in quanto "non c'è 'grazia' di Dio per l'uomo che non sia mediata da Cristo"; rivelativo, poiché è in Cristo che viene offerta la piena comunicazione del mistero di Dio.

Ladaria, nel suo trattato di teologia trinitaria, afferma a tale proposito: "Il nostro punto di partenza sistematico sarà la rivelazione di Dio in Cristo. Non c'è altro modo per giungere al mistero profondo del vero Dio [...] Nella vita di Gesù, nella rivelazione della Trinità economica, ci si dischiude il mistero della Trinità immanente" .

Lo stesso concetto viene ribadito in diversi altri scritti e interventi del teologo spagnolo. Cristo è il "luogo" della rivelazione di Dio: "La dottrina dell'unità divina nella trinità e della trinità nell'unità, che la Chiesa ha sviluppato, è la conseguenza diretta del Dio che Gesù ci ha fatto conoscere" ${ }^{9}$. Tale rivelazione della Trinità in Cristo, spiega il nostro Teologo, non significa tuttavia che il mistero di Dio possa essere minimamente "afferrato" dall'intelletto umano:

La rivelazione di Dio in Gesù, la rivelazione cristiana del Dio uno e trino, è un entrare in contatto con un mistero sempre più grande. [...] Non dobbiamo quindi pensare che la rivelazione di Dio avvenuta in Cristo ci "spieghi" l'essere di Dio o ne renda afferrabile il mistero. [...] La rivelazione cristiana significa in tal modo confrontarsi direttamente con il mistero di Dio ${ }^{10}$.

Questo orizzonte di comprensione garantisce una certa umiltà all'intelligere della teologia, e, al tempo stesso, conferma che in Cristo avviene la piena rivelazione tanto di Dio, quanto dell'uomo.

Oltre a fondare biblicamente e nel solco della Tradizione queste considerazioni, F. L. Ladaria si attiene all'ermeneutica offerta dalla Gaudium et spes, come egli stesso afferma:

$7 \quad$ Ibidem, p. 482 .

8 Il Dio vivo e vero. Il mistero della Trinità, Piemme, Casale Monferrato (Al) 1999, p. 33. Cfr. Cristología del Logos y cristología del Espíritu, pp. 353-354.

$9 \quad$ Il Dio vivo e vero, p. 19.

10 Ibidem, p. 21. 
Il Concilio Vaticano II, in un testo di capitale importanza [...], ha detto che Cristo, rivelando il mistero del Padre e del suo amore, svela anche pienamente l'uomo all'uomo e gli fa nota la sua altissima vocazione (cf. GS 22) ${ }^{11}$.

Cristo, nel suo essere vero Dio e vero uomo, è il rivelatore del Padre e il rivelatore dell'uomo; per tale ragione, per spiegare gli elementi fondamentali dell'antropologia cristiana, il punto di partenza del discorso non può non essere l'evento-Cristo.

La cristologia è radicalmente legata all'antropologia: il Verbo incarnato, infatti, assumendo la carne, ha fatto dell'umano il "luogo" privilegiato della sua espressione. Al tempo stesso, però, è l'antropologia ad essere legata alla cristologia, proprio perché è "in Cristo" che l'uomo non solo scopre, ma riceve la sua vera identità. Si potrebbe addirittura asserire che Dio fa parte della coscienza che l'uomo deve avere di se stesso. La creatura può assumere una vera consapevolezza di sé soltanto nel momento in cui si percepisce "relazionato a" Qualcuno che lo ha creato.

Ladaria individua tre componenti fondamentali per descrivere l'uomo nel suo essere relazionato a Dio. Non si tratta ovviamente di tre tappe cronologicamente distinte, ma di tre aspetti essenziali della condizione creaturale.

Prima di tutto è Dio che, in Cristo, instaura uno stretto rapporto con la sua creatura; e questo indica che la vocazione ultima e definitiva dell'uomo, sempre in Cristo, è quella divina. Scrive Ladaria: "Siamo amati da Dio nel suo Figlio e siamo chiamati a partecipare pienamente della sua vita alla fine dei tempi"12.

Secondo aspetto; questo riferimento a Dio, che determina la vocazione dell'uomo, non elimina la libertà creaturale:

Questa consistenza è necessaria affinché possa realizzarsi questa chiamata, che è rivolta a noi stessi. [...] La condizione creaturale dell'uomo è un determinante fondamentale e totale del suo essere, e deve essere teologicamente considerato nella sua propria consistenza in quanto di fatto orientato alla comunione personale con Dio di cui è contemporaneamente il presupposto necessario ${ }^{13}$.

Terzo aspetto; l'uomo, pur avendo una vocazione divina e una consistenza sua propria, vive l'esperienza del peccato: "Lantropologia teologica deve considerare l'uomo nel suo essere peccatore"14.

Questo profondo legame tra uomo e Dio, pur inverandosi nell'incarnazione del Verbo divino, inerisce nell'essere stesso dell'uomo, sin dalla sua creazione. Afferma ancora la Costituzione pastorale del Concilio Vaticano II: "Adamo,

\footnotetext{
$11 \quad$ Introduzione alla antropologia teologica, p. 10.

12 Ibidem, p. 11.

13 Ibidem, p. 12.

14 Ibidem.
} 
infatti, il primo uomo, era figura di colui che doveva venire"(GS 22). Questo non significa che l'evento-Cristo precede temporalmente la creazione dell'uomo, come se fosse il suo arché: Cristo è l'orizzonte all'interno del quale l'uomo può comprendere se stesso, la sua vera natura, nel suo essere radicalmente (da un punto di vista morale) e ontologicamente (sul piano dell'essere) orientato verso di Lui: Egli è il télos. Afferma Luis Ladaria:

Il Concilio parla dell'uomo alla luce di Cristo, non semplicemente perché in Cristo tutto viene illuminato ma perché nel Figlio incarnato si scopre in ultima analisi chi è e a che cosa è chiamato l'essere umano; non si tratta di una luce che viene dal di fuori ma della stessa realtà della vita di Cristo. [...] Non è Adamo che spiega Cristo, ma Cristo che spiega Adamo. Perciò, solo per mezzo di Cristo possiamo sapere cos'è l'uomo ${ }^{15}$.

Qui si può intravedere una questione già accennata, decisiva per la teologia: l'incarnazione del Verbo divino ha un significato non soltanto soteriologico, ma anche rivelativo: il darsi di Dio è all'insegna del suo dirsi. "Il Verbo si è fatto carne" (Gv 1, 14): questo evento cristologico ha sicuramente una dimensione redentiva (il pro nobis et propter nostram salutem), ma è anche l'espressione massima, l'esegesi, in cui Dio dice Se stesso.

\section{Creazione dell'uomo in Cristo}

Questo discorso, articolato a partire dalla Gaudium et Spes, offre alla riflessione antropologica di Ladaria alcuni elementi fondamentali: prima di tutto Cristo assume un significato ontologico per la vita del credente, non soltanto in ordine escatologico, ma anche protologico: "lui costituisce il modello nella cui sequela e conformazione noi uomini conseguiamo la pienezza, perché fin da principio è il modello a immagine del quale l'uomo è stato creato dal primo istante" 16 .

Fondandosi su alcuni testi scritturistici (1Cor 8, 6; Eb 1, 1-3; Gv 1, 3.10; Col 1, 15-20) il nostro Teologo mette in luce come

a partire dalla presa di coscienza di questo significato salvifico definitivo di Cristo si giunge, nel Nuovo Testamento, all'affermazione della sua mediazione nella creazione, ad attribuirgli un significato protologico. In questo modo si ricavano le estreme conseguenze del significato salvifico di Gesù, nello stesso tempo in cui viene ulteriormente rafforzato ${ }^{17}$.

15 L'uomo alla luce di Cristo nel Vaticano II, in Vaticano II. Bilancio e prospettive, II, a cura di R. Latourelle, Cittadella Editrice, Assisi 1987, pp. 939-951, qui p. 943.

16 Gesù Cristo salvezza di tutti, Edizioni Dehoniane, Bologna 2009, p. 15-16.

$17 \quad$ Antropologia teologica, p. 31. 
La creazione dell'uomo diviene così il presupposto della vocazione di ogni uomo, vale a dire la conformazione a Cristo. Secondo elemento fondamentale è la nuova dignità della natura umana proprio in relazione alla sua stessa assunzione da parte del Verbo divino: "Laffermazione conciliare, però, va oltre. La natura umana di Cristo non solo non viene annientata o assorbita dalla divinità, ma questa natura è stata elevata a una dignità sublime 'anche in noi'. [...] L'umanità viene esaltata con l'assunzione della stessa da parte del Figlio di Dio"18.

Poiché Cristo offre un significato protologico, oltre che quello escatologico, il discorso su cui Ladaria si concentra è il rapporto tra creazione dell'uomo e incarnazione del Verbo divino, soffermando l'attenzione, in particolare, all'evento dell'assunzione. Un teologo spesso citato, proprio per il suo personale contributo su questo argomento, è Karl Rahner, secondo il quale l'incarnazione è la condizione di possibilità della creazione, e non viceversa. Nel suo Corso fondamentale sulla fede ${ }^{19}$, il teologo tedesco arriva a definire l'uomo come la «cifra di Dio»" ${ }^{20}$ egli è "la 'grammatica' - dice Ladaria spiegando Rahner - di una possibile autocomunicazione divina. È il linguaggio che Dio crea per potersi esprimere liberamente e poter comunicare le verità salvifiche il cui contenuto in fondo è lui stesso" "21. Per Rahner, l'uomo è lo strumento migliore, è l'espressione stessa di Dio; l'uomo è Dio che vuole esprimersi fuori di Sé: "L'uomo è quel che nasce - scrive Ladaria, riportando il pensiero di Rahner quando Dio non vuole essere-Dio"22. Più che di "grammatica" o di "cifra", Luis Ladaria preferisce parlare piuttosto di Cristo come "misura" dell'uomo. Scrive infatti: "se l'umanesimo proclama che l'uomo è la misura di tutte le cose, il cristianesimo proclama che c'è ancora una misura dell'uomo, Gesù. [...] È la misura che ci ha posto Dio perché giungiamo alla perfezione di noi stessi”23.

Si può così arrivare così a parlare dell'antropologia come di una cristologia deficiente, proprio per sottolineare che l'uomo può raggiungere la sua pienezza soltanto in Cristo (homo perfectus). È quanto afferma sempre la Gaudium et Spes: "Chiunque segue Cristo, l'uomo perfetto, si fa lui pure più uomo" (GS 41). Scrive Ladaria a tale proposito: "Notiamo che si parla di Gesù come dell'uomo perfetto. Non si tratta soltanto del fatto che sia perfetto uomo, nel senso di uomo

Gesù Cristo salvezza di tutti, pp. 17-18.

K. Rahner, Corso fondamentale sulla fede. Introduzione al concetto di cristianesimo, San Paolo, Cinisello Balsamo (Mi) $2005^{6}$.

Ibidem, pp. 292-295.

Gesù Cristo salvezza di tutti, p. 38.

Ibidem, p. 39.

Ibidem, p. 50. 
completo, come ha affermato la Tradizione sin dagli inizi. Il Concilio Vaticano II ha aggiunto, credo, una nuova sottolineatura: questa umanità completa è perfetta, cioè, è esemplare, paradigmatica" ${ }^{24}$.

Questo, secondo il teologo spagnolo, sottolinea la novità dell'evento-Cristo sotto diversi aspetti: "In primo luogo questa novità significa che solamente in Cristo si rivela il disegno di Dio che era nascosto fin dalla creazione del mondo" 25 . Il fatto che tutto sia stato creato in vista di lui significa che soltanto con l'incarnazione si poteva svelare il progetto di Dio. Scrive Ladaria: "Non solo il disegno di Dio si è rivelato quando è arrivata la pienezza dei tempi, ma l'uomo si è opposto fin dal primo istante ai disegni di Dio"26, e questo vuol dire che Cristo ha portato l'umanità da un livello di schiavitù, in cui essa si era posta nel peccato, ad un livello che realizza la sua vocazione ultima. Il Teologo aggiunge un altro elemento: "Leffettiva novità della venuta di Cristo è che lui in persona porta la salvezza, la sapienza, la giustizia, la santificazione e la redenzione" ${ }^{27}$ : questo farci-Dio di Cristo è avvenuto attraverso il suo farsi-uomo.

Proprio in virtù di questo, Ladaria afferma in modo lapidario: "Luomo non è misura di Cristo, ma è Cristo la misura dell'uomo. Lantropologia deve attingere i propri criteri definitivi dalla cristologia e non da altro"28.

È in Cristo, nel suo essere vero Dio e vero uomo, che tra cristologia e antropologia si apre un vero e proprio circolo ermeneutico: se da un lato, infatti, l'antropologia presuppone la cristologia (così come la creazione presuppone l'incarnazione), dall'altro, spiega il teologo spagnolo: "la cristologia presuppone un'antropologia, sebbene la superi e la sottometta a critica. Nell'incontro con Cristo l'uomo sa già qualcosa su di sé, e se Cristo può illuminare la sua condizione, è perché la condivide"29.

\section{Creaturalità come filiazione}

La domanda che ci si può porre, alla luce di quanto affermato, è la seguente: cosa Cristo rivela all'uomo dell'uomo? La prima risposta che propone Ladaria è: la filiazione divina. Proprio in quanto Figlio, Cristo rivela all'uomo la sua realtà ontologica. Scrive Luis Ladaria:

\footnotetext{
$24 \quad$ Introduzione alla antropologia teologica, p. 28.

25 Gesù Cristo salvezza di tutti, p. 21.

26 Ibidem.

27 Ibidem, p. 22.

28 Ibidem, p. 25.

29 Ibidem, p. 26.
} 
Nella sua rivelazione di Dio come proprio Padre, Gesù si mostra in modo molto peculiare come Figlio. Nella molteplicità dei titoli cristologici del Nuovo Testamento, quello di 'Figlio' o 'Figlio di Dio' è stato considerato, già fin dai primi tempi e in tutta la tradizione della Chiesa, come quello che meglio ha rappresentato l'identità ultima di Gesù. [...] La rivelazione dell'uomo che Gesù fa nel rivelare Dio come Padre è, di conseguenza, la rivelazione della chiamata alla filiazione divina, come partecipazione alla filiazione originale di Gesù ${ }^{30}$.

Essere figli nel Figlio non è una formula allusiva, ma è un dato oggettivo, ontologico e rivelativo, che accade "per partecipazione". Ecco allora che il primo elemento dell'identità dell'uomo ad essere rivelato da Cristo è la figliolanza. Il Nuovo Testamento esprime in modo evidente che la relazione filiale di Gesù con il Padre non coincide con la nostra, eppure l'uomo non ne rimane estraneo, ma partecipa "alla filiazione originale e irripetibile di Cristo" ${ }^{31}$. In questo orizzonte di comprensione la formula figli nel Figlio assume il suo significato più autentico. La partecipazione all'essere-Figlio di Cristo non nega lo statuto ontologico della filiazione umana, né vuole esprimere una subordinazione della seconda al primo, ma dice che solo in Cristo e nello Spirito Santo (cfr. Rm 8, 15; Gal 4, 6) ogni uomo può rivolgersi a Dio come Abbà, Padre. E questa comprensione della filiazione umana viene chiaramente affermata anche dalla Gaudium et Spes: "Tale e così grande è il mistero dell'uomo, che chiaro si rivela agli occhi dei credenti, attraverso la rivelazione cristiana [...] Cristo è risorto, distruggendo la morte con la sua morte, e ci ha donato la vita, affinché, figli nel Figlio, esclamiamo nello Spirito: Abbà, Padre" (GS 22) ${ }^{32}$.

Questo passaggio rivela un evidente cristocentrismo, che però sa farsi tuttavia "mediazione" cristologica: in Cristo (figli nel Figlio) ci rivolgiamo al Padre. Senza avere la pretesa di dare definizioni, si potrebbe parlare in Ladaria di un evidente cristocentrismo trinitario, che non solo elimina ogni possibile cristomonismo, ma che inserisce l'uomo all'interno della stessa vita divina.

\section{Filiazione come fraternità}

Cristo rivela all'uomo dell'uomo un altro aspetto fondamentale: la sua chiamata ad una solidarietà con tutti gli uomini:

Questo è il vincolo di unità più forte fra gli uomini che possiamo concepire. In questa comune vocazione in Cristo a essere membri del unico corpo si trova il più profondo vincolo di solidarietà fra gli uomini. La nostra relazione filiale con il

\footnotetext{
$30 \quad$ Ibidem, pp. 45-46.

$31 \quad$ Ibidem, p. 46.

32 Cfr. Ibidem, p. 47.
} 
Padre, in unione con Gesù, si può vivere solo nella fraternità fra gli uomini. In Gesù, pertanto, si fonda una nuova relazione fra noi, proprio quella della fraternità ${ }^{33}$.

La fraternità, qui, oltre ad essere intesa nel suo significato sociologico, ha un fondamento chiaramente cristologico e trinitario. Scrive Luis Ladaria:

Lamore ha un punto di partenza che si trova in Dio stesso e nell'amore che Egli ci ha mostrato inviandoci Gesù. Da questo punto si deve necessariamente partire quando si tratta dell'amore dal punto di vista cristiano. [...] È [...] necessario ritornare sempre alla sorgente ${ }^{34}$.

La "misura" dell'amore di Dio è stata rivelata in Cristo, e in particolare nell'evento della crocefissione: "è proprio questo amore di Cristo fino alla morte sulla croce che ci fa conoscere fino a che punto siamo amati da Dio Padre" 35 . Questa "misura senza misura", oltre a offrire la salvezza, esprime l'essere stesso di Dio: "Dio è amore" $(1 \mathrm{G} v 4,16)$. Tale amore è universale: "Non possiamo dimenticare che da un punto di vista cristiano la vocazione di tutti gli uomini alla filiazione divina in Cristo crea un profondo vincolo di fraternità dal quale nessuno può essere escluso"36.

È l'esperienza che l'uomo fa dell'amore di Dio (e dunque che Dio è amore) a fondare e argomentare l'amore fra le persone: è dalla certezza di essere figlio di Dio che posso relazionarmi agli altri come fratello. Al tempo stesso, però, l'amore fraterno è ciò che permette all'uomo di rivolgersi, singolarmente ed anche ecclesialmente, a Dio come figlio, tanto che, se la reciprocità interumana dovesse venire a mancare, non si potrebbe neanche invocare Dio come Padre; per esprimere questo forte legame e vincolo tra amore di Dio e amore del prossimo, Luis Ladaria cita la dichiarazione conciliare Nostra aetate: "Non possiamo invocare Dio Padre di tutti gli uomini, se ci rifiutiamo di comportarci da fratelli verso alcuni tra gli uomini che sono creati a immagine di Dio. Latteggiamento dell'uomo verso Dio Padre e quello dell'uomo verso gli altri uomini suoi fratelli sono tanto connessi che la Scrittura dice, chi non ama, non conosce Dio $(1 \mathrm{G} v 4,8)$ " 37 (NA 5).

È nell'amore verso gli altri che gli occhi dell'uomo si dischiudono ed egli può fare esperienza dell'amore di Dio. Afferma il teologo spagnolo: "Senza l'amore del prossimo non esiste amore di Dio [...]. Lamore di Dio implica l'amore al

\footnotetext{
$33 \quad$ Ibidem, pp. 47-48.

34 Amore di Dio e del prossimo, "Gregorianum" 91/2 (2010), pp. 219-239, qui p. 220.

35 Ibidem, p. 221.

36 Dignitatis personae. Alcuni elementi di antropologia, "Studia Moralia" 47/2 (2009), pp. 339-353, qui p. 350.

37 Nostra aetate, 5.
} 
prossimo e non si compie davvero senza la pratica concreta di esso" ${ }^{38}$. Tale atteggiamento di uscita dell'uomo, da se stesso verso gli altri, non è un atteggiamento etico, ma è epifania dell'essere stesso dell'uomo, creato a immagine e somiglianza della Trinità. Per questo motivo Ladaria afferma lapidariamente: "l'uomo non può trovare la sua perfezione se non nella dedizione agli altri" 39 . Qui per "perfezione" si intende il raggiungimento della pienezza dell'umanità, destino che non è applicato all'uomo dall'esterno, ma "risponde a ciò che fin dall'eternità era nel piano di Dio ed ha incominciato ad attuarsi nel primo istante della creazione" ${ }^{40}$.

Lamore dell'uomo verso i suoi fratelli, pur essendo radicato ontologicamente in lui, non scaturisce da una sua personale attitudine: anche l'amore con cui gli uomini si amano tra loro è "dono", e dunque viene da Dio. E questo non perché la Trinità funga da semplice esempio da imitare, e neanche soltanto perché Dio è l'origine (essendo Amore) di tutto ciò che è, ma perché Egli è la dinamica stessa che spinge l'uomo ad amare:

Questa risposta umana è solamente possibile perché Dio stesso ci fa amare e ci muove ad amare. Lamore non viene da noi. [...] Essendo la natura divina amore, dandoci l'amore Dio ci dà qualcosa di se stesso, appunto il suo Spirito, che è il mezzo mediante il quale l'amore di Dio è effuso in noi. L'amore di Dio, cioè Dio stesso, è entrato in noi fino al centro del nostro essere. [...] Soltanto perché abbiamo in noi l'amore di Dio, concretizzato nel dono dello Spirito Santo, possiamo noi uomini a nostra volta amare ${ }^{41}$.

Il fatto, però, che l'amore che spinge ogni uomo ad andare incontro ai suoi fratelli, ad essere radicalmente proiettato verso di loro, venga da Dio, non significa che non ci sia una piena collaborazione in questa propensione da parte dell'uomo. Luis F. Ladaria, ripercorrendo il contributo di diversi autori medievali, mostra come Dio e uomo non sono concorrentit ${ }^{42}$.

\section{Conclusioni}

Straordinariamente chiaro è il ritmo interno alla riflessione di Luis F. Ladaria sull'antropologia teologica. Egli parte dall'orizzonte tracciato dal Concilio Vaticano II e muove il suo pensiero a partire dai diversi studi e pronunciamenti magisteriali. Per parlare dell'uomo non si può non parlare di Dio, ma tale

\footnotetext{
$38 \quad$ Amore di Dio e del prossimo, art. cit., p. 231.

39 Gesù Cristo salvezza di tutti, p. 48.

40 Ibidem.

41 Amore di Dio e del prossimo, art. cit., pp. 234-235.

42 Cfr. Ibidem, pp. 236-237.
} 
asserzione non cade in una deducibilità consequenziale, ma si avvalora di un profondo spessore teologico e si invera attraverso una necessaria mediazione cristologica: è in Cristo, infatti, che viene svelato all'uomo tanto il mistero di Dio, quanto il mistero di se stesso. La centralità cristologica, in questo orizzonte di comprensione, non ha un significato "esemplare", vale a dire come se egli fosse un modello etico, ma ha un valore pienamente ontologico, che trova nell'essere vero Dio e vero uomo del Verbo incarnato la sua condizione di possibilità. Una riflessione di antropologia teologica, dunque, non può non tenere conto e non può non essere determinata dalla cristologia e dalla trinitaria.

Un altro elemento fondamentale che caratterizza il pensiero di Ladaria sull'uomo è che questa intima relazione con Cristo e con il mistero della Trinità trova la ragione del suo essere non tanto in un'accezione puramente escatologica, ma in una protologica: la creazione dell'uomo, a immagine e somiglianza del suo Creatore ( $\mathrm{cfr}$ Gen 1, 26-27), avviene già in vista di Cristo, di colui che ha sviluppato pienamente l'essere-uomo. E questo è indice di un principio basilare della teologia, che in Ladaria emerge in modo evidente ed esplicito: è il telos a spiegare l'arché. In altre parole: è l'Incarnazione a spiegare la creazione, è l'ultimo Adamo a spiegare il primo Adamo. E' alla luce dell'escatologia che si può penetrare all'interno del vero senso della protologia. Questo principio rende evidente come Cristo funga da prezioso perno non solo soteriologico, ma anche rivelativo, in quanto offre una particolare teo-logica alla riflessione ecclesiale, un metodo che trova proprio in lui la sua peculiarità.

Avendo Cristo come nucleo fondamentale del discorso, la realtà antropologica e quella trinitaria non rischiano di confondersi o giustapporsi tra loro, ma tengono conto, sempre richiamandosi al principio dell'analogia (il quale afferma che in tanta somiglianza è sempre maggiore la dissomiglianza), di un'unità che sappia sempre garantire l'intrinseca distinzione che la caratterizza.

Proprio per questo il passaggio dal piano divino a quello umano viene realizzato, nell'essere vero Dio e vero uomo del Verbo incarnato, in modo tale che ciascuno dei due possa ritrovare nella relazione con l'altro la specificità sua propria. E questo non solo sul piano concettuale, ma anche su quello linguistico. Il che garantirebbe, per fare un esempio, nel parlare di tematiche quali l'amore, la comunione, la relazione, la reciprocità, di poter passare dal livello teologico a quello antropologico, senza correre il rischio di applicare ad uno le caratteristiche dell'altro: errore in cui è facile imbattersi, e in cui diverse riflessioni teologiche sono cadute.

La relazione, in Cristo, tra uomo e Dio e, a partire dalla rivelazione della Trinità, quella degli uomini tra loro trovano nell'intimità del rapporto tra Padre e Figlio e Spirito Santo la loro condizione ermeneutica e ontologica di possibilità. Per questo motivo, per un approfondimento sistematico dell'antropologia 
teologica di Ladaria, la ricerca non può essere rivolta in modo esclusivo a quei testi che trattano dell'uomo, dal tema della sua creazione a quello della sua vocazione, ma è necessario tener conto anche dei diversi altri suoi scritti che toccano, con sottolineature più cristologiche e trinitarie, la realtà di Dio.

In questo saggio non si è voluto presentare il pensiero del nostro Autore nella sua globalità, ma solo indicare alcune linee e coordinate essenziali della sua riflessione sull'uomo.

Con la certezza che la grandezza di un pensatore non si può misurare dai nuovi contenuti che vuole presentare, ma dall'articolazione e dalla coerenza interne alla sua riflessione, si può sicuramente affermare che il grande contributo di Luis F. Ladaria stia proprio nel ritmo, squisitamente teologico, della sua antropologia, che riesce a tenere conto simultaneamente tanto della cristologia quanto della trinitaria. Tale apporto trova nelle diverse asserzioni presenti nei documenti del Concilio Vaticano II il suo orizzonte di comprensione, tanto da poter presentare il nostro Autore tra coloro che hanno forgiato un'antropologia teologica tipicamente postconciliare: è qui che l'uomo trova la sua piena e perfetta identità in Cristo, e non nell'una o nell'altra ipotesi sociologica, che oggi sembra essere la più accreditata, la più "di moda", e dunque seguita, e domani tenderà, con la massima naturalezza, ad essere obliata e superata. C'è qualcosa, nel DNA dell'uomo, che non cambia, e che trova via di espressione, nello scorrere della storia e nell'evoluzione della conoscenza, nella definitività dell'evento cristologico.

Parole chiavi: Ladaria, antropologia, ermeneutica, mediazione, filiazione, immagine, fraternità.

\section{Bibliografia:}

1. Commissione Teologica Internazionale, Teologia - cristologia - antropologia, (10.1982), in Enchiridion Vaticanum 8, Bologna 2004'.

2. Góźdź K., Chrysto-antropologia abpa Luisa F. Ladarii, „Studia Bobolanum” 1(2011).

3. Ladaria L. F., Amore di Dio e del prossimo, "Gregorianum" 91/2 (2010).

4. Ladaria L. F., Antropologia teologica, Piemme, Casale Monferrato (Al) 2002.

5. Ladaria L. F., Cristología del Logos y cristología del Espíritu, "Gregorianum" 61 (1980).

6. Ladaria L. F., Dignitatis personae. Alcuni elementi di antropologia, "Studia Moralia" 47/2 (2009).

7. Ladaria L. F., Gesù Cristo salvezza di tutti, Edizioni Dehoniane, Bologna 2009.

8. Ladaria L. F., Il Dio vivo e vero. Il mistero della Trinità, Piemme, Casale Monferrato (Al) 1999. 
9. Ladaria L. F., Introduzione alla antropologia teologica, Gregorian \& Biblical Press, Roma 2011 .

10. Ladaria L. F., L'uomo alla luce di Cristo nel Vaticano II, in Vaticano II. Bilancio e prospettive, II, a cura di R. Latourelle, Cittadella Editrice, Assisi 1987.

11. K. Rahner, Corso fondamentale sulla fede. Introduzione al concetto di cristianesimo, San Paolo, Cinisello Balsamo (Mi) $2005^{6}$. 